\title{
How Protein Labeling Applying
}

\author{
Bian Xinyu ${ }^{1, *, \dagger}$, Fan Sun ${ }^{2, \dagger}$, Zhixuan $\mathrm{Xu}^{3, \dagger}$ \\ ${ }^{1}$ Reading College, Nanjing University of Information Science \& Technology, 210044, Nanjing, China \\ ${ }^{2}$ Shanghai Soong Ching Ling School, 201703, Shanghai, China \\ ${ }^{3}$ Dulwich International High School-Suzhou, 215000, Suzhou, China \\ ${ }^{\dagger}$ These authors contributed equally to this paper
}

\begin{abstract}
In recent years, biosensing has played an irreplaceable position in scientific fields as well as our daily lives. A biosensor is an instrument that is sensitive to biological substances and converts its concentration into electrical signals for detection. It is an analysis tool or system composed of immobilized biologically sensitive materials as identification elements, appropriate physical and chemical transducers and signal amplification devices, and it has the functions of both a receiver and a converter. Pregnancy test sticks, blood glucose testing, etc. in life are all potential biosensors. The main point of this paper is to introduce several main techniques of biosensing for better understanding, like ELISA, fusion proteins, fluorescent probe and others. In the future, the bright future for them is also worth looking forward to if more researches and experiments can be processed in this area, for example, in the detection and treatment of cancer, medical technology can be further improved.
\end{abstract}

\section{Introduction}

Biological researches usually require the use of molecular labels covalently linked to the target protein to facilitate the detection or purification of the labeled protein, which is the general use of the protein labeling technique. Different labeling methods result in different molecules being covalently bound to the target protein. For some researches, specialized protein modifications are required to increase molecular mass, enlarge solubility for storage, or create a new functional group that can be targeted in the following stage. To study this labeling technique further, a review has been presented about protein labeling. The primary organization of this paper is divided into three parts, the comparison between enzyme linked immunosorbent assay (ELISA) and Western Blot, between fusion proteins and immune-fluorescence and between the fluorescent probe and quantum dots separately.

\section{Protein labeling strategy: ELISA and Western Blot}

The current mainstream biosensors include ELISA, fluorescent protein, western blot, etc., which are used for quantitative detection. The high efficiency and operability of ELISA detection make it one of the widely used detection techniques.

Western blot (WB) is a common method to detect and analyze proteins. It is based on a technique that transfers proteins from electrophoresis to membrane by electrophoretic separation and displays them specifically on the membrane. Agarose or polyacrylamide gel electrophoresis is one of the core technologies of gene manipulation. It can be used to isolate, identify and purify DNA fragments. This technique is simple and rapid and has become the technical basis of many common molecular biology research methods, for example, DNA recombination, DNA nucleotide sequence analysis, DNA restriction enzyme analysis, restriction enzyme mapping. When a molecule is placed in an electric field, it will move to the corresponding electrode at a certain speed, which is the migration speed of electrophoretic molecules under the action of the electric field. This mobility is called electrophoretic mobility. It is proportional to the electric field strength and the net charge carried by the electrophoretic molecule itself. That is to say, the higher the electric field intensity, the more net charges the electrophoretic molecules carry and the faster the migration speed, and vice versa. Due to the use of nonreactive stable supporting media, such as agarose gel and polyacrylamide gel, to reduce convective motion, the mobility of electrophoresis is inversely proportional to the coefficient of friction. It is well known that friction coefficient is a function of molecular size, polarity and medium viscosity. Therefore, according to the size, composition or shape of molecules and the amount of net charge, various components in protein or nucleic acid molecular mixture can be separated by electrophoresis. Under physiological conditions, the phosphate group in the phosphate backbone of nucleic acid sugar is in the ionic state. In this sense, DNA and RNA polynucleotide chains can be called polyanions. Therefore, when nucleic acid molecules are placed in an electric field, they migrate to the positive electrode. Due to the repetitive structure of

* Corresponding author: ma803008@student.reading.ac.uk 
the glycerophosphate backbone, the same amount of double-stranded DNA has almost the same net charge, so they can migrate to the positive electrode at the same speed. Under a certain electric field, the mobility of DNA molecule, that is, the mobility of electrophoresis depends on the size and configuration of the nucleic acid molecule. DNA molecules with smaller molecular weights migrate faster than those with larger molecular weights. This is the basic principle of DNA fragments separated by gel electrophoresis [1].

Samples were prepared and loaded into the gel. In the process of electrophoresis, the protein with a negative charge moves to the anode with a positive charge. To further analyze these proteins, the process of transferring them to the membrane is called imprinting. After transfer, the membrane is blocked to prevent unnecessary membrane protein interactions in the following steps. To visualize the proteins of interest, the first-order proteinspecific antibody detection membrane is usually used, and then the labeled second-order antibody is used for detection. The film was imaged and the results were analyzed.[2]

By adding a separate marker solution to a hole in the gel, it can not only estimate the antibody interaction of a specific protein but also estimate the size of the protein. The separation of the gel depends not only on the size but also on the molecular charges, hydrophobic regions and denaturation. The experimental settings can be varied, which is most suitable for specific exploration. When analyzing the results, the load variation between lanes and the transfer rate between bolts must be considered. Besides, the nonlinear relationship of the generated signal in the range of sample concentration is also an aspect to be considered when interpreting the results. [3] The results of the leukocyte test depend on three important factors: the ability of the antibody to bind specific proteins, the intensity of interaction and the concentration of protein itself. Also, the specificity and low cross-reactivity of target binding are important characteristics. WB results are not always easy to explain because protein size may differ from theoretical weight due to post-translational modifications (such as glycosylation) or interactions with other proteins. However, WB is a quite common method, and almost all available commercial antibodies have been verified by this method.[4]

Western blotting is a technique for the analysis of antigens and antibodies. It has the following advantages.

1). The time of incubation and washing was significantly shortened.

2). To be used for multiple analysis and identification, multiple copies can be made at the same time.

3). The results can be displayed in the form of a chromatogram and can be stored for a long time.

4). Immunoprobe can be used to erase the probe by reducing the $\mathrm{pH}$ value and replace it with a second probe for analysis and detection.

5). The wet immobilized matrix membrane is flexible and easy to operate.

6). The immobilized biomacromolecules can approach all kinds of immune probes uniformly, and the pore size will not block them.
7). The amount of reagent needed for Western blot analysis was less. ELISA is a quantitative measurement method for specific substances. It connects antibodies to a specific enzyme for identification and measurement.

There are four major types of ELISA. Which are Direct ELISA (antigen-coated plate; screening antibody), Indirect ELISA (antigen-coated plate; screening antigen/antibody), Sandwich ELISA (antibody-coated plate; screening antigen) and Competitive ELISA (screening antibody). No matter it is direct or indirect ELISA, both of them have to fix the antigen on the plate.[5] When the antigen is added to the plate, it will be incubated at 37 degrees for one hour. The second step is very important, all unbound sites on the plate will be blocked with reagents such as animal protein. This step ensures that there will not be any non-specific antibodies bind to the plate later. After adding the buffer in the last step, wash the plate again and incubate for another hour after adding the antibody. Some enzymes that can change the color will be added to the direct ELISA. Compared with other detection methods, direct ELISA is simpler and more efficient. And it also contains the disadvantages of high reaction cost and low sensitivity. The steps of indirect ELISA are basically the same as those of direct ELISA, but compared with direct ELISA, the antibody is washed first and then added. Indirect ELISA uses two kinds of antibodies [6]. "A primary detection antibody that sticks to the protein of interest and a secondary enzyme-linked antibody complementary to the primary antibody." Compared to direct ELISA, indirect ELISA is cheaper and more sensitive. But indirect ELISA has a disadvantage, it can easily cause a chain reaction [7]. "Once the coating step is complete, the plates are washed with phosphate buffer saline (PBS), then buffered/blocked with bovine albumin (BSA). The buffer washes are carried out for at least 1-2 hours at room temperature. Finally, the plate is washed with PBS once again before the addition of the antigen." The disadvantage of Sandwich ELISA is that they require more time and cost. The last type of ELISA is competitive ELISA. Two types of antibodies are both added in the competitive ELISA, which leads to the competition of substrates. Its advantage is that this ELISA can more accurately measure smaller and more diverse antigens.

ELISA has many medical applications. For example, it is usually used for food allergies tests, pregnancy tests and HIV tests. The ELISA test usually has two test zones. One is for control, which makes sure that the test kit is working. The second test zone shows whether it is positive or negative. However, a second test is necessary for the HIV test, because the antibodies may haven't been secreted yet, which may lead to inaccuracy [7].

\section{Protein labeling strategy: fluorescent labels}

The attachment of fluorescing parts to proteins or other biomolecules is a common but useful strategy widely used for us to research protein structure and function in a better way. These fluorescent proteins have a broad range of 
applications, including immunochemistry, fluorescence in situ hybrids, cell tracing, receptor labeling, and fluorescence spectroscopy, etc. Two methods are introduced here, that are mainly used in the bioresearch area, one is the fusion protein method, and another is the immune-fluorescence method.

\subsection{Fusion protein}

The fusion protein is a kind of special protein which combined by mixing two or more genes that have been originally coded for separate proteins. The single or multiple polypeptides translated from this fusion gene have functional properties that are derived from each original protein. Among these proteins, the recombinant fusion protein is an important variable, which is created by recombinant DNA technology, and they are playing an irreplaceable role in the use of biological research or therapeutics area. And recombination technology, in which genes or gene fragments are spliced together for the formation of a gene fusion, has become an attractive tool for biotechnology, in particular in protein labeling [8].

At the mention of fusion proteins, an introduction about chimera mutant protein is necessary. Chimera mutant protein usually designates hybrid proteins made of polypeptides that have different functions. Chimeric mutant proteins can easily be prepared in vitro Recombinant, which means by fusing the structural genes of the proteins in a suitable expression vector. There are essentially two types of fusion proteins (Fig. 1) [9].
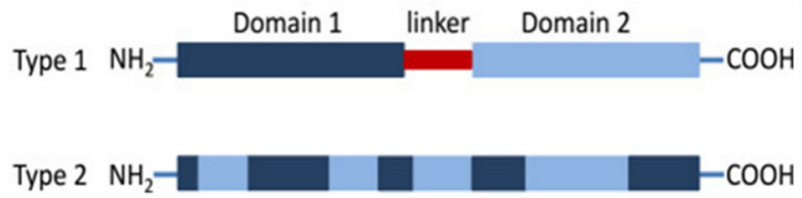

Figure 1. Basic types of fusion proteins.

Besides, chimeric mutant proteins can also be produced naturally when a complex mutation (a chromosomal translocation, tandem duplication, retrotransposition) creates a peculiar coding sequence containing coding sequences' parts from two different genes. Commonly occurring fusion proteins are found in cancer cells, where they may function [10].

Just because of such characteristics of the fusion proteins, there is a research team proposed a method to label antiglobulin test fusion proteins with different fluorophores in mammalian cells. This research shows that different specific fluorophores can be used to label AGT fusion proteins that have different localizations in cells, and the fluorophores can also be used for other applications like multicolor analysis of the dynamic process of labeling and fluorescence resonance energy transfer measurement. Due to the diversity of AGT substrates and the specificity of labeling reactions, this method is convenient in the field of studying protein functions in living cells.

The processes are simple, they synthesized AGT substrates firstly, which is prepared by reacting BG derivative with commercially available N-hydroxy- succinimide esters. And then is the construction of AGT fusion proteins, the AGT mutant here is based on human AGT. Fluorescence Labelling of AGT Fusion Proteins is the final step. All AGT fusion proteins were added to a special solution for transient transfection was performed.

What important are the results they get. AGT fusion proteins show the possibility to label individual fusion proteins through kinds of different fluorophores. In live cells, AGT fusion proteins can be labeled specifically to synthesize fluorophores sequentially. The localization of this kind of fusion protein they researched can be confirmed through mainly two methods, specific targeting sequences and the natural subcellular localization of the fusion position to which the AGT is linked. And the future of the stability of AGT fusion proteins labeled by fluorescence can be tracked in several hours. Besides, what important is that varieties of different fluorophores can be applied to label a single AGT fusion protein for the analysis of multicolor or FRET applications. And these characteristics also make this kind of fusion protein a vital object for fluorescence-based researches of how the protein functions in live cells [11].

\subsection{Immune fluorescence}

Then are a few things about the immune fluorescence method. Immunofluorescence is an important essay that is mainly used on biological samples and its official definition is a procedure that uses antibodies to detect antigens in cellular contexts. Immunofluorescence depends on the specificity of antibodies to the antigen. And it allows researchers to measure whether a particular sample cell expresses the antigen in research. What's more, researchers also can easily determine the target subcellular compartments expressing the antigen by the technology of immunofluorescence when a positive signal is found. Also, immunofluorescence can be applied to cultivating cell lines, tissue sections, or individual cells [12].

After some researches, it is widely known that hemolysis of the Sendai virus can be assayed in the erythrocytes system by poly-L-lysine. Multiple-step experiments can perform easily because of the immobilization of cells with the help of cooperation with lectins and antibodies. These ligands' binding had a remarkable inhibitory effect on viral hemolysis. These subtle molecular rearrangements are a prelude to cell fusion in which disintegration of the viral envelope structure is observed. This process could be characterized by the expression of virus and host-specific antigens with immune fluorescence techniques.

According to these molecular rearranging at the researched site, it is easier to make sense that the dispersal of viral compounds in the membrane. It could be observed by viral antigens' immune fluorescence directly or indirectly that the granular fluorescence shows diffuse staining in the researched position, indicating that viral parts conducted integration and dispersal in the membrane plane [13].

To conclude, both these two techniques have their value in different areas, with no better and worse 
differences. For fusion protein expression, it can be easily detected in protein labeling researches and separated for purifying, but it also may affect the structure of the original protein and destroy its natural function if wrong using. For the immunofluorescence method, like most fluorescence techniques, a significant problem that shouldn't be ignored is photobleaching, which can be controlled by increasing the density of fluorescence or limiting the intensity of light.

\subsection{Fluorescent probe}

The fluorescent probe, also called fluorophores or simply fluors, respond distinctly to light compared to other molecules. As shown below, a photon of excitation light is absorbed by an electron of a fluorescent particle. In excited via absorption of light energy to a higher energy state. Also called an excited state, the energy of the excited state, which cannot be sustained for long delays or decreases. Resulting in the emission of light energy. This process is called fluorescence. To flourish means to emit light via this process of law reform is a molecule that is capable of fluorescence and its ground state.[14] The fluorophore for a molecule is in relatively low energy, stable configuration, and it does not for us. When light from an external source hits a fluorophore, for a molecule, the molecule can absorb the light energy. If the energy absorbed a sufficient. The molecule reaches a higher energy state called an excited state. This process is known as excitation. There are multiple excited states or energy levels of the fluorophore before can attain, depending on the wavelength and energy of the external light source. Since the fluorophore of four is unstable and high energy configurations, it eventually adopts the lowest energy excited state, which is semi-stable.[15] The length of time that the fluorophore for his inexperience states is called the exciting lifetime, and it lasts for a very short time, ranging from ten to the minus fifteen to turn to the minus nine seconds. Next, the fluor for rearranges from the semistable excited state. Back to the ground state and the excess energy is released and admitted as light. The emitted light is of lower energy, and thus, the longer wavelengths than the absorbed light. This means that the color of the light that is emitted is different from the color of the light that has been absorbed.[16] Emission of light returns to the fluorophore, afford to its ground state, the fluorophore, can absorb light energy again and go to the entire process repeatedly. A cyclical fluorescents process can be summarized as one excitation of a fluorophore, or for it through the absorption of light.[17] To a transient, excited lifetime with some loss of energy. And three return of the floor, afford to its ground state, accompanied by the emission of light. The light energy emitted always has a longer wavelength, and the light energy is absorbed due to the energy lost during the transience, excited lifetime, as shown in step two. A fluorophore can repeatedly undergo the fluorescence process, in theory, indefinitely. This is extremely useful because it means that one fluorophore of four molecules can generate a signal multiple times. This property makes fluorescents a very sensitive technique for visualizing microscopic samples. Even a small amount of the stain can be detected. In reality, however, the fluorophore for structural instability during the exciting lifetime, makes it susceptible to degradation. Fluorescent probes with the best photophysical properties have the advantages of high sensitivity and fast response and are important research tools for biological imaging. [18]

Up to now, various fluorescent dyes have been used to construct various types of fluorescent probes. In particular, anthracane-based dyes, such as luciferin, rhodamine and Rodanil, have been developed over the past few years due to their excellent photophysical properties (high quantum yields and extinction coefficients, excellent water solubility and photostability). However, there are still some challenging problems to solve: most dyes containing heteranthracene emit green and red light (500$600 \mathrm{~nm}$ ). In contrast, the near-infrared emission (650$900 \mathrm{~nm}$ ) has obvious advantages, such as less light damage to biological samples, deep penetration of tissues, and less interference from biomolecules to background autofluorescence. Therefore, it is necessary to prepare more near-infrared anthracene dyes. In recent years, heteranthracene nuclei have been modified by extending $\pi$ conjugation or replacing epoxy atoms with other elements, such as $\mathrm{C}$ and $\mathrm{Si}$. They produce some new red emission and near-infrared yellow raw dyes. However, like traditional anthracene dyes, they are still limited by the Stokes shift (usually less than $30 \mathrm{~nm}$ ), which leads to severe fluorescence self-quenching and measurement errors in excitation backscattering. It is well known that the ideal near-infrared dyes for the construction of sensitive imaging probes should not only have high fluorescence quantum yield and large stokes shift, but also have optically tunable groups, which can be easily modified to obtain a variety of reaction-based fluorescent probes. Unfortunately, the number of anthracene dyes that meet the above criteria is still quite limited.

\subsection{Quantum dots}

Quantum dot is a kind of semiconductor material, which is generally spherical or quasi-spherical in diameter of 2$20 \mathrm{~nm}$. The more common quantum dots are composed of IV, II-VI, IV-VI or III-V elements. Specific examples include silicon quantum dots, germanium quantum dots, cadmium sulfide quantum dots, cadmium selenide quantum dots, cadmium telluride quantum dots, zinc selenide quantum dots, lead sulfide quantum dots, lead selenide quantum dots, indium phosphide quantum dots and indium arsenide quantum dots. As a kind of nanometer semiconductor material, quantum dots can emit light with a specific frequency by applying a certain frequency of electric field or light pressure. The frequency of the light will change with the size of quantum dots. By adjusting the size of quantum dots, the frequency of light emitted by them can be within the range of visible light, and the wavelength of light can be directly observed through the change of color. The quantum dots have large Stokes shifts. Another optical property of quantum dots different from organic dyes is the wide Stokes shift, which can avoid the overlap of the emission spectrum and 
excitation spectrum, and is conducive to the detection of fluorescence spectrum signal. Good biocompatibility. After a variety of chemical modifications, quantum dots can be specifically linked, which has low cytotoxicity and little harm to organisms, and can be used for in vivo labeling and detection. Among all kinds of quantum dots, silicon quantum dots have the best biocompatibility. For quantum dots containing cadmium or lead, it is necessary to package the surface of quantum dots before biological application. Quantum dots have a long fluorescence lifetime. The fluorescence lifetime of organic fluorescent dyes is only a few nanoseconds (which is equivalent to the decay time of autofluorescence in many biological samples). The fluorescence lifetime of quantum dots with a direct band-gap can last for tens of nanoseconds (20-50 NS), and that of quantum dots with a quasi-direct bandgap, such as silicon quantum dots, can last for more than $100 \mu \mathrm{s}$.[19] In this way, in the case of light excitation, most of the spontaneous fluorescence has decayed, while the fluorescence of quantum dots still exists. At this time, the fluorescence signal without background interference can be obtained. In a word, QDs have the advantages of wide and continuous excitation spectrum, narrow and symmetrical emission spectrum, adjustable color, high photochemical stability and long fluorescence lifetime. At present, water-soluble quantum dots and oil-soluble quantum dots can be coupled with antibodies, streptavidin and other molecules, which can be used in the fields of biomarker detection, high-throughput coding, in vivo imaging and dynamic tracing.[20] However, the size of water-soluble quantum dots combined with biological macromolecules is large (about $10 \mathrm{~nm} \sim 30 \mathrm{~nm}$ in diameter), which hinders its passage through the cell membrane structure. Therefore, it can only be used for the study of permeabilized cells or extracellular proteins or proteins that can be endocytosed by cells.

\section{Conclusion}

In general, as a relatively mature technology, ELISA has emerged in different branches. The advantages of direct and indirect ELISAs are their high efficiency, speed and low cost. Their operability has become the most extensive household biosensor on the market. For fusion protein expression, it can be easily detected in protein labelling researches and separated for purifying, but it also may affect the structure of the original protein and destroy its natural function if wrong using. For the immunofluorescence method, like most fluorescence techniques, a significant problem that shouldn't be ignored is photobleaching, which can be controlled by increasing the density of fluorescence or limiting the intensity of light. And QDs have the advantages of wide and continuous excitation spectrum, narrow and symmetrical emission spectrum, adjustable colour, high photochemical stability and long fluorescence lifetime.

\section{References}

1. Luo Yunbo. Introduction to food biotechnology, 3rd Edition: China Agricultural University Press, page 27 (2016.08)

2. Burnette WN., "Western blotting": electrophoretic transfer of proteins from sodium dodecyl sulfate-polyacrylamide gels to unmodified nitrocellulose and radiographic detection with antibody and radioiodinated protein A. Anal Biochem. PubMed: 6266278 (1981)

3. N Am J. Western Blotting technique, theory, and trouble shooting:Mahmood $\mathrm{T}$ et al., Western blot: technique, theory, and trouble shooting. PubMed: 23050259 DOI: 10.4103/1947-2714.100998 (2012)

4. Biotechnology. The first article describing electrophoretic transfer of proteins to membrane:Towbin $\mathrm{H}$ et al., Electrophoretic transfer of proteins from polyacrylamide gels to nitrocellulose sheets: procedure and some applications. (1992)

5. National Center for Biotechnology Information. PubChem Bioassay Record for AID 686974 (2001) Source:

https://pubchem.ncbi.nlm.nih.gov/bioassay/686974.

6. Application Center for Biotechnology Information. PubChem Bioassay Record for AID 686974 (2019) Source: https://pubchem.ncbi.nlm.nih.gov/bioassay/686974.

7. Barnett, C. ELISA Applications. News-Medical Life Science. PubMed: 1422008. Source: https://www.news-medical.net/life-sciences/ELISAApplications.aspx

8. Uhlén, M., Forsberg, G., Moks, T., Hartmanis, M., \&amp; Nilsson, B.. Fusion proteins in biotechnology. (2004, November 11). Source: http://www.sciencedirect.com/science/article/abs/pii /095816699290164E

9. Chimeric protein. (2011, May 3). Source: https://www.sciencedirect.com/topics/medicine-anddentistry/chimeric-protein

10. Strohl, W.. Chimeric genes, proteins. (2006) Source: https://www.sciencedirect.com/science/article/pii/B9 780128096338061999

11. Hub: Labeling of fusion proteins with Synthetic fluorophores in LIVE Cells: 10.1073/pnas.0401923101. (2004, May 24). Source: https://sci-

hub.ren/https://www.pnas.org/content/101/27/9955.s hort

12. Principle of immunofluorescence. (2001, April 11) Source:

https://www.sinobiological.com/category/principleof-if

13. Bächi, T., Eichenberger, G., \&amp; Hauri, H. Sendai virus HEMOLYSIS: Influence of lectins and analysis by Immune fluorescence. (2015, Augest 11). Source: https:/www.sciencedirect.com/science/article/abs/pi i/0042682278904580 
14. Fluorescent probe. (2002, July 23). Source: https://www.sciencedirect.com/topics/chemistry/fluo rescent-probe

15. Immunofluorescences. (2001, February 21). Source: https://www.thermofisher.com/cn/zh/home/lifescience/protein-biology/protein-biology-learningcenter/protein-biology-resource-library/pierceprotein-methods/fluorescent-probes.html

16. Fluorescent probes. (2002, June 25) Source: https://www.thermofisher.com/cn/zh/home/lifescience/protein-biology/protein-biology-learningcenter/protein-biology-resource-library/pierceprotein-methods/fluorescent-probes.html

17. Fu, Y., \&amp; Finney, N. Small-molecule fluorescent probes and their design. (2018, August 14). Source: https://pubs.rsc.org/en/content/articlelanding/2018/r a/c8ra02297f

18. Quantum dot. (April 14, 2003), Source: https://www.sciencedirect.com/topics/materialsscience/quantum-dot

19. Design resource molecule. (2006, December 11). Source: https://www.sigmaaldrich.com/technicaldocuments/articles/materialsscience/nanomaterials/quantum-dots.html

20. Berger, M. What are quantum dots? (2019, October 15). Source: https://www.nanowerk.com/what_are_quantum_dot s.php 